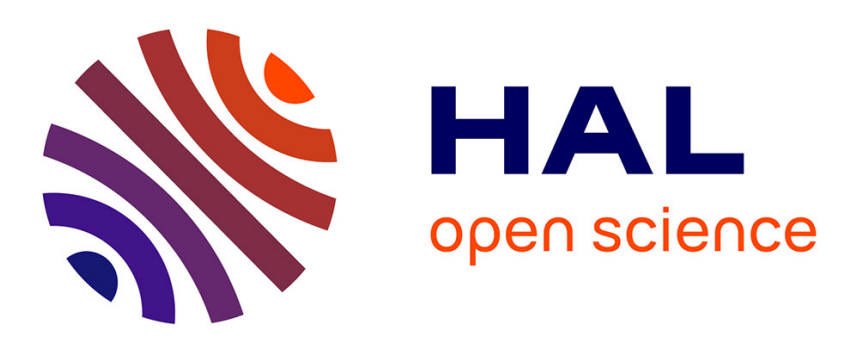

\title{
Mitigating Primary Emulation Attacks in Multi-Channel Cognitive Radio Networks: A Surveillance Game
}

Duc-Tuyen Ta, Nhan Nguyen-Thanh, Patrick Maillé, Philippe Ciblat, van Tam Nguyen

\section{- To cite this version:}

Duc-Tuyen Ta, Nhan Nguyen-Thanh, Patrick Maillé, Philippe Ciblat, van Tam Nguyen. Mitigating Primary Emulation Attacks in Multi-Channel Cognitive Radio Networks: A Surveillance Game. GLOBECOM 2016 - 2016 IEEE Global Communications Conference, Dec 2016, Washington, United States. 10.1109/GLOCOM.2016.7841989 . hal-01713280

\section{HAL Id: hal-01713280 \\ https://hal.science/hal-01713280}

Submitted on 20 Feb 2018

HAL is a multi-disciplinary open access archive for the deposit and dissemination of scientific research documents, whether they are published or not. The documents may come from teaching and research institutions in France or abroad, or from public or private research centers.
L'archive ouverte pluridisciplinaire HAL, est destinée au dépôt et à la diffusion de documents scientifiques de niveau recherche, publiés ou non, émanant des établissements d'enseignement et de recherche français ou étrangers, des laboratoires publics ou privés. 


\title{
Mitigating primary emulation attacks in multi-channel cognitive radio networks: A surveillance game
}

\author{
Duc-Tuyen $\mathrm{Ta}^{1}$, Nhan Nguyen-Thanh ${ }^{1}$, Patrick Maille ${ }^{2}$, Philippe Ciblat ${ }^{1}$, and Van-Tam Nguyen ${ }^{1,3}$ \\ ${ }^{1}$ LTCI, CNRS, Tlcom ParisTech, Universit Paris Saclay, 75013, Paris, France \\ 2 Telecom Bretagne/IRISA, Institut Mines-Telecom, Rennes, France \\ ${ }^{3}$ Department of EECS, University of California at Berkeley, USA
}

\begin{abstract}
Primary User Emulation Attack (PUEA), in which attackers emulate primary user signals causing restriction of secondary access on the attacked channels, is a serious security problem in Cognitive Radio Networks (CRNs). An user performing a PUEA for selfishly occupying more channels is called a selfish PUEA attacker. Network managers could adopt a surveillance process on disallowed channels for identifying illegal channel occupation of selfish PUEA attackers and hence mitigating selfish PUEA. Determining surveillance strategies, particularly in multichannel context, is necessary for ensuring network operation fairness. In this paper, we formulate a game, called multi-channel surveillance game, between the selfish attack and the surveillance process in multi-channel CRNs. The sequence-form representation method is adopted to determine the Nash Equilibrium (NE) of the game. We show that performing the obtained NE surveillance strategy significantly mitigates selfish PUEA.
\end{abstract}

\section{INTRODUCTION}

Cognitive radio (CR) is introduced as a solution to improve spectrum utilization by enabling secondary access to licensed spectrum. Discovering spectrum holes is therefore essential. To explore the spectrum opportunities, there are two approaches: spectrum sensing and database-driven. In the spectrum sensing approach, the primary users' activity is explored by measuring the spectrum environment, while in the database-driven approach, the information of spectrum usage for CR users is provided by a database server. Compared to the databasedriven approach, the spectrum sensing approach is cheaper and more flexible for a wide range of networks [1]. However, spectrum sensing faces a serious security risk: Primary User Emulation Attack (PUEA) [2]-[8]. In PUEA, the attacker transmits an emulated Primary User (PU) signal which could lead to disallowed state on the attacked channels (i.e., the channels which spectrum sensing system claims to be busy after the sensing period). The attacker therefore gains exclusive spectrum right. Depending on goals, PUEA can be categorized into two types: selfish and malicious. A malicious PUEA targets at ruining the operation of networks, hence it is similar to the jamming attack or the denial-of-service attack. A selfish PUEA, however, aims at selfishly occupying the attacked channel for data transmission. In that sense, selfish PUEA is associated with an illegal benefit degrading the fairness of CR Networks (CRNs) and possible interference threating to primary systems. In this study, we therefore focus on how to mitigate the selfish PUEA on CNRs.

Several earlier works have investigated the issues of selfish PUEA. Most of them adopt detection methods based on additional information, such as the locations of both primary and secondary users [2] or the frequency deviation feature of FM signal [4] to identify the PUEA attackers. However, those methods are only applicable on special cases where added information, or the imperfection of attacked signals is available. The other works applied the game-theoretical framework to analyze the risk of PUEA [5]-[10] since there are the opposing objectives between the attacker and the network manager. In [5], the authors formulate a non-cooperative multistage game between a selfish PUEA attacker and a secondary node on the data transmission phase. A dogfight spectrum game between a PUEA attacker and a CR user is formulated in [6]. In that work, PUEA attacking signals are treated as jamming signals and channel hopping is proposed as a solution for mitigating PUEAs. However, there is still vulnerability if the attacker conducts multiple channel attacks.

A successful selfish PUEA is usually followed by selfishly using of the attacked channel by the attacker. Therefore, it is possible to determine the illegal accessing in any communication link through the users identification. In our previous works [7], [8], we have proposed a surveillance process to mitigate the influence of selfish PUEA by monitoring data traffic at the beginning of the data frame on an occupied channel. To determine the surveillance strategy of the network manager, we use a game-theoretic approach to formulate the relation between the attack and the surveillance process in a single channel. The best strategies of the attacker and the network manager are figured out in closed-form, as a Nash equilibrium (NE) point. However, CRNs usually work on multiple frequency bands, and because of the rapid expansion of software-defined radio, the attacker can launch multichannel selfish PUEA. For such a case, a sequential monitoring plan can be used, however, at the cost of long surveillance time. In this paper, we therefore consider the multi-channel surveillance process to mitigate the influence of the selfish PUEA in CRNs.

The multi-channel surveillance model is more complicated but more realistic than the single-channel model. We formulate 
the relation between the selfish PUEA and the surveillance process as a two-player game in extensive form and consider the Nash Equilibrium (NE) for the surveillance strategy. Note that this approach can be extended to mitigate the influence of malicious PUEA or unknown-attacking PUEA in CRNs.

Typically, the network manager observes the attacker's action only indirectly, through the sensing results. Hence, the formulated game is an incomplete and imperfect information game. Finding a Nash equilibrium solution in this game is more complicated than in perfect-information games. We employ the sequence-form representation method [11], [12] instead of the conventional (benchmark) strategic-form representation method [13] to determine the best strategy for the defender and the attacker. We prove that the sequenceform representation is much more efficient than the strategicform representation method. We then analyze and interpret the impact of the system parameters includes the PU's presence probabilities, the network demand, as well as the penalty factor on the obtained NE strategies.

\section{SySTEM MODEL}

We call the attacker, the representative of PUEA users and the defender, the resource manager of the network which monitors the traffic on disallowed channels to detect selfish attackers. Let $N$ be the number of available channels, $M$ be the maximum number of channels that the attacker can attack and $L$ be the maximum number of channels that the defender can monitor.

The timing frame for network operation is the same as our previous work (Figure 1 in [7]). Before the transmission (data phase), a sensing phase, possibly followed by a surveillance phase, is carried out. For each channel, because of the nonideal sensors, two possible sensing results could be obtained: "allowed", and "disallowed". An attacker can implement a selfish PUEA by transmitting the emulated primary user signals during the sensing time. We assume the sensors cannot distinguish the emulated and authentic primary signals. Consequently, the PUEA will not be detected in the sensing time. During a PUEA, the attacker cannot know the true status of the primary user on the attacked channel since it cannot sense for PU signal at the same time. This means the PUEA attacker conducts the attack in a blind condition regarding the primary signal status. Considering the defense against selfish PUEA threats, we assume that a fixed format data frame is used for exchanging data with all CR users including selfish users. The format contains the identifying information of a user such as the medium access control address. Therefore, $\mathrm{CR}$ users can be identified by observing transmitted signals in data time. Channel surveillance processes which are conducted on monitored secondary access channels can identify the selfish attackers if they are presented. Once a selfish attacker has been detected, punishments such as network isolation or bandwidth limitation can be adopted to penalize the attacker. This surveillance process is assumed to be implemented by the resource manager.
TABLE I

NOTATIONS (AT THE $i^{\text {th }}$ CHANNEL)

\begin{tabular}{|c|c|}
\hline Notation & Meaning \\
\hline$\pi_{i}$ & The presence probability of PU. \\
\hline$p_{N}^{i}$ & $\begin{array}{l}\text { The probability that the sensor answers disallowed channel } \\
\text { when the attacker does not attack. }\end{array}$ \\
\hline$p_{A}^{i}$ & $\begin{array}{l}\text { The probability that sensor answers disallowed channel when } \\
\text { the attacker attacks. }\end{array}$ \\
\hline$\rho_{N}^{i}$ & $\begin{array}{l}\text { The probability that the channel is not used by the PU while } \\
\text { the sensor claims disallowed and the attacker does not attack. }\end{array}$ \\
\hline$\rho_{A}^{i}$ & $\begin{array}{l}\text { The probability that the channel is not used by the PU while } \\
\text { the sensor claims disallowed and the attacker attacks. }\end{array}$ \\
\hline$C_{A}^{i}$ & The implementing cost of the selfish PUEA. \\
\hline$G_{A}^{i}$ & The using gain of selfish PUEA attacker at one data frame. \\
\hline$C_{S}^{i}$ & The monitoring cost of the resource manager at the channel. \\
\hline$G_{M}^{i}$ & $\begin{array}{l}\text { The capturing gain for detecting illegal attack during the } \\
\text { surveillance process of data frame. }\end{array}$ \\
\hline$P^{i}$ & The penalty value for being captured at the channel. \\
\hline
\end{tabular}

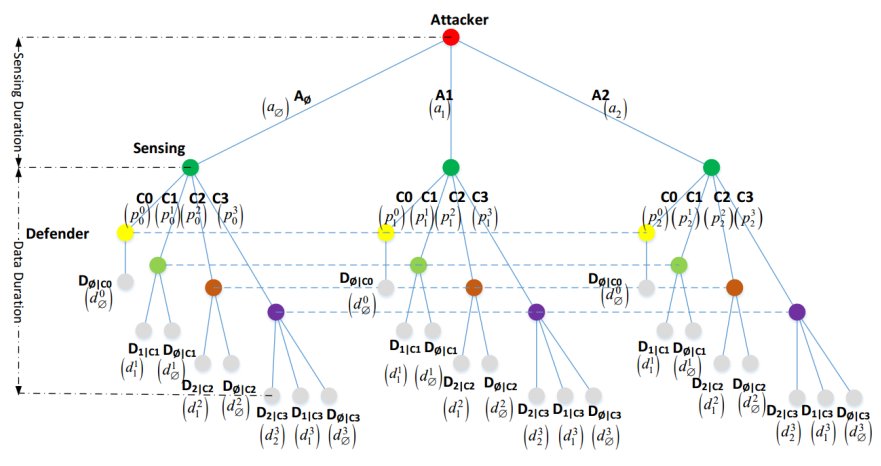

Fig. 1. Two-channel surveillance game

Before the analysis, we summarize the specific notations that will be used throughout the paper (Table I).

\section{GAME FORMULATION}

We formulate a two-player game in extensive form, called the multi-channel surveillance game (MSG), to present the relationship between the multi-channel surveillance process and the selfish PUEA in CRNs. There are two players: Attacker (player 1) who represents selfish PUEA attackers, and Defender (player 2) who represents the resource manager. Figure 1 illustrates the MSG game for a CRN with two channels $(N=2)$, the attack capability of the attacker $M=1$ and the monitoring capability of the defender $L=1$.

1) Strategies: We denote by $A_{\emptyset}, A_{1}, A_{2}, \ldots, A_{K_{1}}$ the actions of the attacker, where $A_{\emptyset}$ corresponds to the action No Attack and $A_{i}$ corresponds to the action Attack of a nonempty subset of available channels. The attacker has $\left(K_{1}+1\right)$ actions leading to its pure strategy set $\Sigma_{A}$.

$$
\Sigma_{A}=\left\{A_{\emptyset}, A_{1}, A_{2}, \ldots, A_{K_{1}}\right\}
$$

where $K_{1}=\sum_{i=1}^{\min (M, N)}\left(\begin{array}{c}N \\ i\end{array}\right)$.

Let $\mathbf{C}$ be the set of the sensing result of all channels and $\mathbf{C}$ includes $2^{N}$ elements. For a sensing result $C_{k} \in \mathbf{C}$ $\left(k=0, \ldots, 2^{N}-1\right)$, let $\left|C_{k}\right|$ be the number of the disallowed channels. We denote by $D_{\emptyset \mid C_{k}}, D_{1 \mid C_{k}}, D_{2 \mid C_{k}}, \ldots, D_{\mid C_{k} \| C_{k}}$ 
the possible actions of the defender in which $D_{\emptyset \mid C_{k}}$ corresponds to the No Surveillance action and $D_{j \mid c_{k}}$ corresponds to the Surveillance action of a disallowed channel subset belong $C_{k}$. The pure strategy set of the defender has $K_{2}$ actions leading to its pure strategy set $\Sigma_{D}$.

$$
\Sigma_{D}=\bigcup_{k=0}^{2^{N}}\left\{D_{\emptyset \mid C_{k}}, D_{1 \mid C_{k}}, D_{2 \mid C_{k}}, \ldots, D_{\left|C_{k}\right| \mid C_{k}}\right\}_{C_{k} \in \mathbf{C}}
$$

and $K_{2}=1+\sum_{m=1}^{\min (N, L)} \sum_{n=1}^{m}\left(\begin{array}{l}m \\ n\end{array}\right) \times\left(\begin{array}{l}N \\ m\end{array}\right)$.

We denote by $\Delta_{A}$ the mixed strategy set of the attacker and $\Delta_{D}$ the mixed strategy set of the defender.

2) Expected payoff: After sensing phase, there are two possible sensing results for $t$ channel: 1) disallowed and 2) allowed. Depending on the action of two players, their payoffs at $t$ channel are presented in Table II. These payoffs are computed by considering the present of $\mathrm{PU}$ which is represented by the values of $\rho_{A}^{i}$ and $\rho_{N}^{i}$.

TABLE II

PAYOFFS OF THE ATTACKER AND THE DEFENDER AT $t$ CHANNEL

\begin{tabular}{l|l|l|l} 
& {$[$ Defender;Attacker $]$} & No Attack & Attack \\
\hline \multirow{2}{*}{ Disallowed } & No Surveillance & {$[0 ; 0]$} & {$\left[0 ;-C_{A}^{t}+\rho_{A}^{t} G_{A}^{t}\right]$} \\
\cline { 2 - 4 } & Surveillance & {$\left[-C_{S}^{t} ; 0\right]$} & $\begin{array}{l}{\left[-C_{S}^{t}+\rho_{A}^{t} G_{S}^{t} ;\right.} \\
\left.-C_{A}^{t}-\rho_{A}^{t} P^{t}\right]\end{array}$ \\
\hline Allowed & No Surveillance & {$[0 ; 0]$} & {$\left[0 ;-C_{A}^{t}\right]$} \\
\hline
\end{tabular}

We denote by $U_{A}^{t, D_{k \mid C_{j}}}$ the obtained payoff of the attacker by performing the Attack of $t$ channel while the defender plays $D_{k \mid C_{j}}$. Similarly, we denote by $U_{D}^{A_{i}, t}$ the obtained payoff of the defender by monitoring $t$ channel while the attacker plays $A_{i}$. For the action pair $\left\{A_{i}, D_{j \mid C_{k}}\right\}$, the payoffs of the defender $U_{D}^{A_{i}, D_{k \mid C_{j}}}$ and the attacker $U_{A}^{A_{i}, D_{k \mid C_{j}}}$ are given by

$$
\begin{aligned}
U_{A}^{A_{i}, D_{k \mid C_{j}}} & =\sum_{t \in A_{i}} U_{A}^{t, D_{k \mid C_{j}}} \\
U_{D}^{A_{i}, D_{k \mid C_{j}}} & =\sum_{t \in D_{k \mid C_{j}}} U_{D}^{A_{i}, t}
\end{aligned}
$$

We denote by $\Omega_{A}$ the expected payoff of the attacker and $\Omega_{D}$ the expected payoff of the defender, respectively. We have

$$
\begin{aligned}
& \Omega_{D}=\sum_{i=1}^{K_{1}} \delta_{a}\left(A_{i}\right) \sum_{j=0}^{2^{N}-1} p_{C_{j} \mid A_{i}} \sum_{k=1}^{\left|C_{j}\right|} \delta_{d}\left(D_{k \mid C_{j}}\right) U_{D}^{A_{i}, D_{k \mid C_{j}}} \\
& \Omega_{A}=\sum_{i=1}^{K_{1}} \delta_{a}\left(A_{i}\right) \sum_{j=0}^{2^{N}-1} p_{C_{j} \mid A_{i}} \sum_{k=1}^{\left|C_{j}\right|} \delta_{d}\left(D_{k \mid C_{j}}\right) U_{A}^{A_{i}, D_{k \mid C_{j}}}
\end{aligned}
$$

where $p_{C_{j} \mid A_{i}}$ is the probability of the sensing result $C_{j}$ under the attacker's action $A_{i}$ and $\delta_{a}\left(A_{i}\right) \in \Delta_{A}$ and $\delta_{d}\left(D_{k \mid C_{j}}\right) \in \Delta_{D}$ are the mixed strategy of action $A_{i}$ and $D_{k \mid C_{j}}$, respectively.

\section{NASH EQUILIBRIUM}

For the MSG game, we explore the Nash equilibrium (NE) point, i.e., the point where each player has selected a best response (BR) strategy to other players' strategies. The BR are the strategies on which the player gains the highest payoff given other players' strategies. A NE strategy may be a "pure" or a "mixed" strategy. To determine the NE point, two approaches are considered: 1) the conventional strategic-form representation and 2) the sequence-form representation.

\section{A. Strategic-form representation}

We first consider the conventional strategic-form representation, which is based on the Harsanyi transformation [13] and the Lemke-Howson (L-H) algorithm [14]. The Harsanyi transformation models all possible actions of a player, which are affected by the other players' actions and the nature choices. For the MSG game, however, the method results in an exponential increment in the size of the game. In particular, the size of payoff matrix in the MSG game adopting the strategicform representation is $\left(K_{1}+1\right) \times K_{3}$, where $K_{3}$ is given by

$$
K_{3}=\prod_{k=0}^{2^{N}-1}\left(\begin{array}{c}
\left|C_{k}\right| \\
\min \left(M,\left|C_{k}\right|\right)
\end{array}\right)
$$

where $\left(\begin{array}{l}N \\ k\end{array}\right)$ denote a binomial coefficient indexed by $N$ and $k$.

For the case that $M=L=1, K_{3}=K_{3}^{*}=\prod_{k=0}^{N}(k+1)^{\left(\begin{array}{c}N \\ k\end{array}\right)}$. It means that, the payoff matrix is $3 \times 12$ if $N=2$ and $5 \times\left(5 \times 12^{6}\right)$ if $N=4$. It is significantly larger when $M$ and $L$ bigger than 1 . Consequently, it is very complicated to find the NE points of the game for the large $N$.

\section{B. Sequence-form representation}

In game theory, an extensive form game includes the information about the sequencing of players' possible moves, the chance moves, payoffs for both players at the leaves and the information set at the decision nodes. If the game is perfect recall, i.e. each player remembers its' earlier moves, each node has a unique path from the root. Such a game can be represented in the sequence-form where a sequence is defined as a string listing the action choices of a particular player. In detail, for each node $h$ of player $i$, we define $\sigma_{h}$ as the sequence and $C_{h}$ as the set of choices of player $i$ at $h$. For each choice $c \in C_{h}$, the corresponding sequence of $i$ is $\sigma_{h} c$. Hence, the set of sequences $\boldsymbol{\Sigma}_{i}$ for player $i$ is given by

$$
\boldsymbol{\Sigma}_{i}=\{\emptyset\} \cup\left\{\sigma_{h} c \mid h \in H_{i}, c \in C_{h}\right\}
$$

where $H_{i}$ is the set of node of player $i$.

Since the MSG game is a perfect recall game, we adopt the sequence-form representation to solve the game. The trick here is that we consider the sensing results as the elements of the attacker's sequence. The sequence strategy set of the attacker then is

$$
\begin{aligned}
\boldsymbol{\Sigma}_{A}^{s e q} & =\left\{\sigma_{A i}, i=1 \ldots K_{4}\right\} \\
& =\left\{\emptyset, A_{\emptyset}, A_{1}, \ldots, A_{K_{1}}, A_{\emptyset, C_{0}}, A_{\emptyset, C_{1}}, \ldots\right\}
\end{aligned}
$$

where $K_{4}=1+\left(K_{1}+1\right)+\left(K_{1}+1\right) \times 2^{N}$.

The corresponding sequence strategy set of the defender is

$$
\begin{aligned}
\boldsymbol{\Sigma}_{D}^{s e q} & =\left\{\sigma_{D j}, j=1 \ldots\left(K_{2}+1\right)\right\} \\
& =\left\{\emptyset, D_{\emptyset \mid C_{k}}, D_{1 \mid C_{k}}, \ldots, D_{\left|C_{k}\right| \mid C_{k}}, \ldots\right\}_{C_{k} \in \mathbf{C}}
\end{aligned}
$$


The mixed strategy for the sequence-form representation is presented by the probability of each sequence in the sequence strategy set. Let $\Phi_{A}$ and $\Phi_{D}$ denote the mixed sequence strategies for the attacker and the defender, respectively. From the definition of the sequence strategy set, we have

$$
\begin{aligned}
& \Phi_{D}^{s e q}=\left\{\phi_{d}^{i}\right\}_{i=1 \ldots\left(K_{2}+1\right)} \\
& \Phi_{A}^{s e q}=\left\{\phi_{a}^{j}\right\}_{j=1 \ldots K_{4}}
\end{aligned}
$$

where $\phi_{a}^{i}$ is the probability of the attacker's $i^{\text {th }}$ sequence, $\phi_{d}^{j}$ is the probability of the defender's $j^{\text {th }}$ sequence.

The relation between these mixed strategies is called the realization plan. By default, for an empty sequence, the probability is 1 . For any node $h$, the mixed strategy of the sequence at $h$ is the sum of all mixed strategies from $h$. Therefore, we obtain the realization plans for the defender and the attacker:

$$
\left\{\begin{array}{l}
\phi_{d}(\emptyset)=1 \\
\phi_{d}\left(D_{\emptyset \mid C_{k}}\right)+\sum_{i=1}^{\left|C_{k}\right|} \phi_{d}\left(D_{i \mid C_{k}}\right)=1 \forall C_{k} \in \mathbf{C} \\
0 \leq \phi_{d}^{i} \leq 1, i=1 \ldots K_{2}
\end{array}\right.
$$

and

$$
\left\{\begin{array}{l}
\phi_{a}(\emptyset)=1 \\
\phi_{a}\left(A_{\emptyset}\right)+\sum_{i=1}^{K_{1}} \phi_{a}\left(A_{i}\right)=1 \\
\sum_{i=0}^{2^{N}-1} \phi_{a}\left(A_{\emptyset, C_{i}}\right)=\phi_{a}\left(A_{\emptyset}\right) \\
\vdots \\
\sum_{i=0}^{2^{N}-1} \phi_{a}\left(A_{K_{1}, C_{i}}\right)=\phi_{a}\left(A_{K_{1}}\right) \\
0 \leq \phi_{a}^{i} \leq 1, i=1 \ldots K_{4}
\end{array}\right.
$$

In general, these realization plans can be re-written using the following matrix form.

$$
\left\{\begin{array} { l } 
{ \mathbf { E } \Phi _ { A } = \mathbf { e } } \\
{ \Phi _ { A } \geq 0 }
\end{array} , \quad \text { and } \quad \left\{\begin{array}{l}
\mathbf{F} \Phi_{D}=\mathbf{f} \\
\Phi_{D} \geq 0
\end{array},\right.\right.
$$

where $\mathbf{E}$ and $\mathbf{F}$ are called the constraint matrices, and $\mathbf{e}$ and f are vectors in which the first element is 1 , and the other elements are 0 .

As defined above, each leaf of the game tree corresponds to a pair of sequences. Hence, for a pair of the sequence strategies $\left(\sigma_{A i}, \sigma_{D j}\right)$, there are 3 cases: i) if a pair of sequences is ended at a leaf, the payoffs are computed by multiplying (3) and (4) with the corresponding probability of the change move leads to this leaf, ii) if a pair of sequences does not correspond to a leaf, the payoffs are zero and iii) if a pair of sequences corresponds to the leaves and consist the chance moves or the information set, the payoff is then the sum over all leaves that define the given pair of sequences.

Let $\Pi_{A}$ and $\boldsymbol{\Pi}_{D}$ denote the payoff matrix of the attacker and the defender in the sequence-form representation. The expected payoffs of the attacker $\Omega_{A}$ and the defender $\Omega_{D}$ therefore are computed by

$$
\begin{aligned}
& \Omega_{A}=\Phi_{A}^{\mathbf{T}} \boldsymbol{\Pi}_{A} \Phi_{D} \\
& \Omega_{D}=\Phi_{A}^{\mathbf{T}} \boldsymbol{\Pi}_{D} \Phi_{D}
\end{aligned}
$$

An equilibrium is a pair $\left(\Phi_{A}, \Phi_{D}\right)$ of mutual best responses. In particular, if the realization plan $\Phi_{D}$ is fixed, then $\Phi_{A}$ is the best response to $\Phi_{D}$ if and only if it is an optimal solution of the linear program

$$
\begin{array}{cl}
\underset{\Phi_{A}}{\operatorname{maximize}} & \Phi_{A}^{\mathbf{T}}\left(\boldsymbol{\Pi}_{A} \Phi_{D}\right) \\
\text { subject to } & \mathbf{E} \Phi_{A}=\mathbf{e} \\
& 0 \leq \Phi_{A} \leq 1
\end{array}
$$

The dual form of linear program (18) is as follows.

$$
\begin{array}{cl}
\underset{\mathbf{p}}{\operatorname{minimize}} & \mathbf{e}^{T} \mathbf{p} \\
\text { subject to } & \mathbf{E}^{T} \mathbf{p} \geq \boldsymbol{\Pi}_{A} \Phi_{D} \\
& \Phi_{A}^{\mathbf{T}}\left(-\boldsymbol{\Pi}_{A} \Phi_{D}+\mathbf{E}^{T} \mathbf{p}\right)=0
\end{array}
$$

A similar program is established for the attacker strategy.

In conclusion, the problem of finding a Nash equilibrium can be formulated as follows:

$$
\begin{array}{ll}
\text { find } & \mathbf{p}, \mathbf{q}, \Phi_{A}, \Phi_{D} \\
\text { subject to } & \mathbf{E}^{T} \mathbf{p} \geq \boldsymbol{\Pi}_{A} \Phi_{D}, \quad \mathbf{F}^{T} \mathbf{q} \geq \boldsymbol{\Pi}_{D}^{T} \Phi_{D} \\
& \Phi_{A}^{\mathbf{T}}\left(-\boldsymbol{\Pi}_{A} \Phi_{D}+\mathbf{E}^{T} \mathbf{p}\right)=0 \\
& \Phi_{D}^{\mathbf{T}}\left(-\boldsymbol{\Pi}_{D}^{T} \Phi_{A}+\mathbf{F}^{T} \mathbf{q}\right)=0 \\
& \mathbf{E} \Phi_{A}=\mathbf{e} \\
& \mathbf{F} \Phi_{D}=\mathbf{f} \\
& \Phi_{A} \geq 0 \\
& \Phi_{D} \geq 0
\end{array}
$$

The value of $\mathbf{p}, \mathbf{q}, \Phi_{A}, \Phi_{D}$ that satisfies the constraints in (20) can be found through the Linear Complementary Programing (LCP) [15] by introducing the non-negative vector $\mathbf{z}=\left(\Phi_{A}, \Phi_{D}, \mathbf{p}^{\prime}, \mathbf{p}^{\prime \prime}, \mathbf{q}^{\prime}, \mathbf{q}^{\prime \prime}\right)^{T}$ where $\mathbf{p}^{\prime}, \mathbf{p}^{\prime \prime}$ and $\mathbf{q}^{\prime}, \mathbf{q}^{\prime \prime}$ are non-negative vectors of the same dimension as $\mathbf{p}=\mathbf{p}^{\prime}-\mathbf{p}^{\prime \prime}$ and $\mathbf{q}=\mathbf{q}^{\prime}-\mathbf{q}^{\prime \prime}$. Furthermore, we let

$$
\mathbf{M}=\left[\begin{array}{cccccc}
0 & -\boldsymbol{\Pi}_{\mathbf{A}} & \mathbf{E}^{\mathbf{T}} & -\mathbf{E}^{\mathbf{T}} & 0 & 0 \\
-\boldsymbol{\Pi}_{\mathbf{D}} \mathbf{T} & 0 & 0 & 0 & \mathbf{F}^{\mathbf{T}} & -\mathbf{F}^{\mathbf{T}} \\
-\mathbf{E} & 0 & 0 & 0 & 0 & 0 \\
\mathbf{E} & 0 & 0 & 0 & 0 & 0 \\
0 & -\mathbf{F} & 0 & 0 & 0 & 0 \\
0 & \mathbf{F} & 0 & 0 & 0 & 0
\end{array}\right]
$$

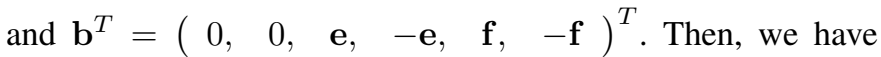
the LCP problem as follows:

$$
\begin{array}{ll}
\text { find } & \mathbf{z} \\
\text { s.t } & \mathbf{M z}+\mathbf{b} \geq 0 \\
& \mathbf{z}^{\mathbf{T}}(\mathbf{M z}+\mathbf{b})=0 \\
& \mathbf{z} \geq 0
\end{array}
$$

The LCP problem above could be solved by the Lemke algorithm [11], [12], [15]. The main idea of the Lemke algorithm is to apply the pivoting operation in the complementary problem. A more detailed description can be found in [15]. Since a feasible solution at least exists for the formulated LCP problem [11], hence from the solution of (20) we achieve the solution of (22). This solution is the NE point of the game. We have the following propositions.

Proposition 1: In the MSG game, the payoff matrix's size in the sequence-form representation is much smaller than the payoff matrix's size in the strategic-form representation. 


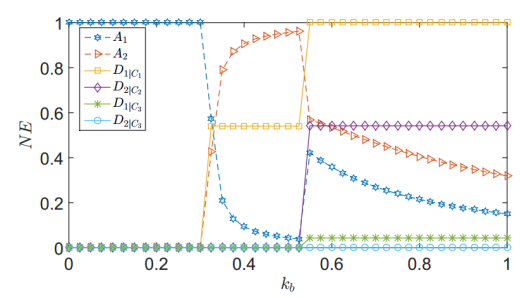

(a) $k_{C}=0.4$

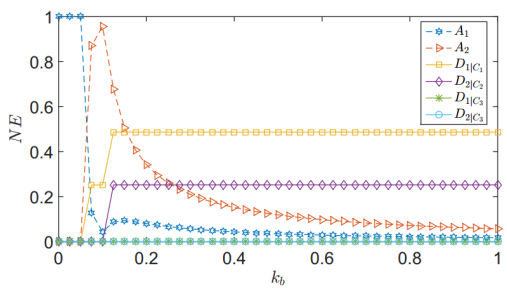

(b) $k_{C}=3$

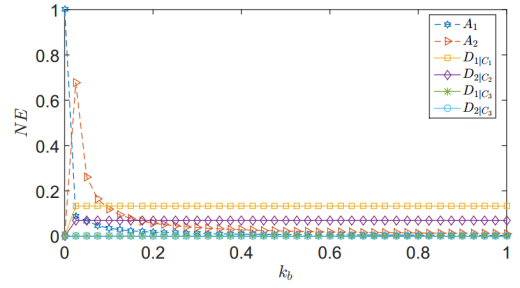

(c) $k_{C}=10$

Fig. 2. The NE strategy of the MSG game for $N=2$, with channel characteristics $\pi_{1}=0.2$ and $\pi_{2}=0.5$.

Proof: We observe the payoff matrix in the sequenceform representation is much smaller than one in the strategicform because $K_{4} \ll K_{3}$. In particular, the payoff matrix in the sequence-form is linear in the size of the game whereas the payoff matrix in the strategic-form is generally exponential. For example, we consider for a CRN with the number of available channels $N=4$ where the attack capability of the attacker and the surveillance capability of the defender $M=L=1$. The size of the corresponding payoff matrix is $86 \times 48$, which is much smaller than one with the strategicform representation $\left(5 \times\left(5 \times 12^{6}\right)\right)$.

Remark 1: Below are some results from Proposition 1.

- The strategy space of the sequence-form representation is exponentially smaller than the strategy space of the strategic-form representation. Since the two methods operate similarly [11], [12], the run time of each algorithm depends on the size of the input. Thus, it is exponentially faster to run the Lemke algorithm on the sequence-form than the LH algorithm on the strategic-form.

- Since the sequence-form representation is much more compact than the strategic-form representation, we therefore adopt the sequence-form representation approach to determine the NE strategy of the MSG game.

Proposition 2: If $L \geq N$, the NE point of the MSG game is unique and corresponds to the combination result of $N$ independent single-channel surveillance games (results in our previous work [7]), each with one channel in the set of available channels.

Proof: (sketch) We consider the CRN with $N$ available channels. When $L \geq N \mathrm{~m}$ the defender can perform to surveillance on all disallowed channels. In this case, it is equivalent to perform single-channel surveillance game of all disallowed channels independently. For each single-channel surveillance game, the NE point is unique and has been figured out in [7]. Therefore, the NE point of the MSG game with $L \geq N$ is unique and could be determined by combining $N$ unique $\mathrm{NE}$ points of $N$ single-channel surveillance games.

\section{Numerical Results}

In order to analyze the impact of system parameters to the NE point of the MSG game, the numerical computations are conducted in Matlab with the Gambit toolbox [16] for game theory. We also assume the sensing system adopts the energy detection method and the average SNRs of the primary signal received at sensor are $-10 \mathrm{~dB}$. The false alarm probability is $P_{f}=0.1$ and the number of samples in the energy detector is $N_{\text {sample }}=1500$. The detection probability $\left(P_{d}\right)$ is then computed through the Constant false alarm rate (CFAR) criterion. To focus on the NE strategy of the game, we assume there is no difference between channels in the attacking costs and in the monitoring gains, and only consider the most significant case $G_{A}^{i}>C_{A}^{i} \forall i=1 \ldots N$ (i.e., the using gain is higher than the attacking cost of a channel).

We introduce the parameters: i) The penalty factor $k_{C}$ : the ratio between the penalty and the using gain $k_{C}=P^{i} / G_{A}^{i}$, ii) the network demand $k_{b}$ : the ratio between the surveillance gain and the penalty $k_{b}=G_{S}^{i} / P^{i}$. In addition, we denote by $k_{A}$ the ratio between the attacking cost and the using gain (i.e., $\left.k_{A}=C_{A}^{i} / G_{A}^{i}\right), k_{S}$ the ratio between the monitoring cost and the using gain (i.e., $k_{S}=C_{S}^{i} / G_{A}^{i}$ ).

We first consider the CRN with difference cases of channel numbers $N$. Table III shows the computational time to determine the NE point of the MSG game by using the sequenceform method and the strategic-form method. The numerical programs are conducted on a Dell Precision M6700 laptop with Intel Core i7 CPUs $2.6 \mathrm{GHz}$. For $N=2$, two methods use the same run time to determine the NE strategy of the game. For $N=3$, however, the sequence method method is much faster than the strategic-form method. For $N>3$, strategicform method is unable to provide results while the sequenceform method is feasible. We therefore adopt the sequence-form method to determine the NE strategy of the MSG game.

TABLE III

THE COMPUTATION TIME TO DETERMINE THE NE POINT.

\begin{tabular}{c|cccc} 
& $N=2$ & $N=3$ & $N=4$ & $N=5$ \\
\hline Strategic-form & $2 \mathrm{~s}$ & $1960.7 \mathrm{~s}$ & $\infty$ & $\infty$ \\
Sequence-form & $2 \mathrm{~s}$ & $32.4 \mathrm{~s}$ & $11564 \mathrm{~s}$ & $\sim 12 \mathrm{~h}$
\end{tabular}

Next, for illustrating the effect of parameters on NE strategy of the MSG game, we consider a CRN with two available channels $(N=2)$, the attack capability of attacker $M=1$ and the monitoring capability of the defender $L=1$. We assume that $k_{A}=0.2, k_{S}=0.1, \pi_{1}=0.2$ and $\pi_{2}=0.5$.

Fig. 2a, Fig. 2b and Fig. 2c present the NE strategy of the MSG game for low penalty $\left(k_{C}=0.4\right)$, medium penalty $\left(k_{C}=2\right)$ and high penalty $\left(k_{C}=10\right)$, respectively. To provide a better view, we only plot the NE strategies of the attack actions and the surveillance actions. We observe that 


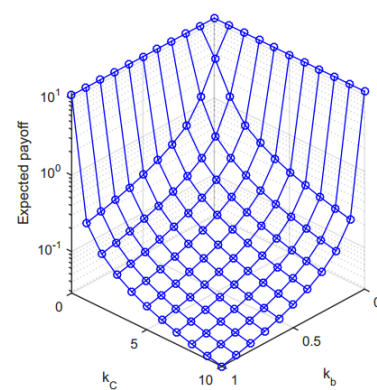

(a) $\Omega_{D}^{(N E)}-\Omega_{D}^{U n i S}$

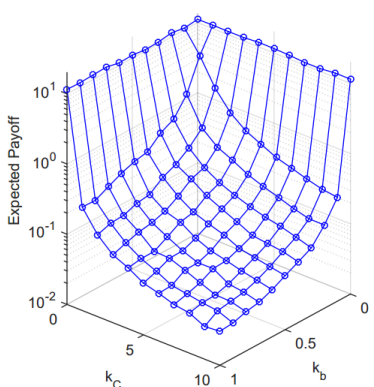

(b) $\Omega_{D}^{(N E)}-\Omega_{D}^{(\text {Rand })}$
Fig. 3. The differences on defender's expected payoffs: (a) between NE and uniform surveillance (UniS) strategies, (b) between NE and random (Rand) strategies.

for each the penalty factors $k_{C}$, the distribution of NE points is separated into three regions along with the increase of the network demand $k_{b}$. First, when the network demand $k_{b}$ is low, the defender does not need to monitor disallowed channels. Consequently, the attacker could perform the attack to the best channel (channel with low operation of PUs). Second, when the network demand $k_{b}$ is medium, the attacker has a trend of moving the attack from the best channel to the worst channel. The reason of this trend is that the defender performs its surveillance on the best channel due to the increase of its spectrum demand. Third, when the network demand $k_{b}$ is very high, the defender increase the surveillance rate at the both channels. As a result, the attacker has a corresponding response by adjusting its attacking rate on the both channels. For each region, the strategy of defender depends on the relation between the network demand $k_{b}$ and the penalty factor $k_{C}$. For a fixed penalty factor $k_{C}$, the defender performs monitoring the disallowed channel with a constant probability for each region. In addition, the size of each region also depends on the penalty factor $k_{C}$ where the last region corresponding with high network demand $k_{b}$ is enlarged with penalty factor values. The results mean that the NE strategies of the MSG game are affected by both the penalty factor and the network demand. We conclude that in order to reduce the influence of PUEA, the CRNs should set a high penalty.

Figure 3 shows the differences on the defender's expected payoffs when the defender plays (a) NE and uniform surveillance strategies, and (b) NE and random strategies. The uniform surveillance strategy means that all disallowed channels are uniformly monitored, and the random strategy means that all possible actions are randomly performed.

For the low network demand $k_{b}$ or the low penalty $k_{C}$, the monitoring gain is smaller than the monitoring cost. If the defender performs to surveillance the disallowed channels, its expected payoff will be a negative value. For the high penalty factor $k_{C}$ or the high network demand $k_{b}$, the NE strategy is the BR for the defender. Therefore, the defender's expected payoff at NE strategy is higher than those at the other strategies. We concluded that the NE strategy is efficient to mitigate the selfish PUEA in multi-channel CRNs.

\section{CONCLUSION}

We have discussed the multi-channel surveillance process to deal with the selfish PUEA in multi-channel cognitive radio networks. Performing a multi-channel surveillance process on disallowed channels help to identify selfish PUEA attacker. The relation between attack strategies and the surveillance process has been formulated through an extensive-form game. Sequence representation method has been used for obtaining Nash equilibrium. The numerical results have showed the influence of the network demand and the penalty factor on controlling NE strategies and the effectiveness of using NE on mitigating PUEA. Besides, the introduced method has a more efficient computational time compared to the conventional one. We will generalize this method to deal with other PUEA attacker types such as malicious and unknown-attacking type.

\section{ACKNOWLEDGMENT}

This work has been supported by French Ministry of Industry and European CATRENE in CORTIF Project.

\section{REFERENCES}

[1] P. Murty, "SenseLess: A Database-Driven White Spaces Network," IEEE Transactions on Mobile Computing, vol. 11, no. 2, pp. 189-203, Feb 2012.

[2] R. Chen and et al, "Defense against Primary User Emulation Attacks in Cognitive Radio Networks," IEEE Journal on Selected Areas in Communications, vol. 26, no. 1, pp. 25-37, Jan 2008.

[3] — , "Toward secure distributed spectrum sensing in cognitive radio networks," IEEE Communications Magazine, vol. 46, no. 4, pp. 50-55, April 2008.

[4] S. Chen and et al, "Hearing Is Believing: Detecting Wireless Microphone Emulation Attacks in White Space," IEEE Transactions on Mobile Computing, vol. 12, no. 3, pp. 401-411, March 2013.

[5] Y. Tan and et al, "Primary user emulation attack in dynamic spectrum access networks: a game-theoretic approach," IET Communications, vol. 6, no. 8, pp. 964-973, May 2012.

[6] H. Li and Z. Han, "Dogfight in Spectrum: Combating Primary User Emulation Attacks in Cognitive Radio Systems, Part I: Known Channel Statistics," IEEE Transactions on Wireless Communications, vol. 9, no. 11, pp. 3566-3577, November 2010.

[7] N. N. Thanh and et al, "Surveillance strategies against primary user emulation attack in cognitive radio networks," IEEE Transactions on Wireless Communications, vol. 14, no. 9, pp. 4981-4993, Sept 2015.

[8] T. Duc-Tuyen and et al, "Extra-sensing game for malicious primary user emulator attack in cognitive radio network," in 2015 European Conference on Networks and Communications (EuCNC), June 2015, pp. 306-310.

[9] M. Felegyhazi and J. Hubaux, "Game Theory in Wireless Networks: A Tutorial," Computing Surveys, ACM, 2006.

[10] B. Wang and et al, "Game theory for cognitive radio networks: An overview," Computer Networks, vol. 54, no. 14, pp. 2537-2561, 2010.

[11] D. Koller and et al, "Efficient computation of equilibria for extensive two-person games," Games and Economic Behavior, vol. 14, no. 2, pp. 247-259, 1996.

[12] B. von Stengel and et al, Tracing equilibria in extensive games by complementary pivoting. Tilburg University, 1996.

[13] J. Harsanyi, "Games with Incomplete Information Played by "Bayesian" Players, I-III," Manage. Sci., vol. 50, no. 12 Supplement, pp. 1804-1817, Dec. 2004.

[14] C. Lemke and J. Howson Jr, "Equilibrium Points of Bimatrix Games," Journal of the Society for Industrial and Applied Mathematics, vol. 12, no. 2, pp. 413-423, 1964.

[15] R. W. Cottle, J.-S. Pang, and R. E. Stone, The linear complementarity problem. Siam, 1992, vol. 60

[16] R. D. McKelvey and et al, "Gambit: Software tools for game theory," 2014. [Online]. Available: http://www.gambit-project.org. 